\title{
POLÍTICAS PÚBLICAS E GESTÃO DE CULTURA EM CAMPOS DOS GOYTACAZES - RJ
}

\author{
Elisabeth Soares da Rocha* \\ André Luís Almeida Peixoto**
}

\begin{abstract}
Resumo
O presente artigo apresenta uma análise sobre as políticas públicas de cultura no município de Campos dos Goytacazes, com o objetivo de conhecer as reais condições da gestão de cultura no município, assim como a relevância de tais políticas para a sociedade. Por ser considerado um município com grande potencial econômico advindo dos royalties petrolíferos e participações especiais, entende-se, a partir daí, que o município goza de um orçamento privilegiado em comparação com outros municípios no País e, portanto, não vivencia uma condição de restrição orçamentária. Dividido em três partes correlacionadas entre si, este artigo traz primeiramenteum breve histórico sobre o conceito de políticas públicas; em seguida apresenta o funcionamento da gestão de cultura no município no período de 2010 a 2015; e por último apresenta o levantamento da oferta de cultura nos períodos 2010/2011 e 2014/2015, seguindo as considerações finais.Para obtenção de dados reais sobre as ações implementadas pela gestão de cultura do município no período estudado, aplicou-se uma metodologia estatística por meio de um levantamento de toda oferta de cultura realizada pela gestão pública no período já citado. A análise comparativa dos resultados obtidos nos períodos possibilitou compreender qual o modelo de política pública que envolve a cultura no município e verificar que em sua maioria baseiam-se principalmente em realização de shows, voltados para artistas que, na sua maioria, não residem no município e para uma ausência de atividades culturais que buscassem a participação e envolvimento da comunidade local. Tais resultados ainda permitiram observar como as políticas culturais carecem o debate público e político no Município de Campos dos Goytacazes.
\end{abstract}

Palavras-chave: Políticas Públicas; Gestão de Cultura; Campos dos Goytacazes.

* Doutoranda em Educação da Universidade Federal Fluminense (UFF); Docente de Artes do Instituto Federal Fluminense - IFF. E-mail: bethrocha12@gmail.com

** Mestrando em Planejamento Regional e Gestão de Cidades - UCAM. Docente do Instituto Federal Fluminense - IFF. E-mail: andrepeixoto@arqlux.com.br 


\section{Introdução}

O artigo possui como objetivo ampliar a reflexão sobre a importância da cultura fundamentada na ontologia do ser social, com vistas a compreender as relações sociais da produção cultural nesse município a partir das implementações de suas políticas culturais.

Este trabalho está dividido em três partes. A primeira concentrase na revisão do conceito de Políticas Públicas e sua aplicabilidade na Gestão em Cultura; a segunda apresenta um pequeno histórico das Políticas Públicas em cultura e as bases em que se aplicam a Gestão em Cultura no Município de Campos dos Goytacazes; a terceira parte apresenta o levantamento da oferta de eventos culturais apresentada pela gestão municipal de cultura no período de fevereiro de 2010 a janeiro de 2011 e no período de fevereiro de 2014 a janeiro de 2015, cujo objeto consistiu na busca de dados estatísticos necessários para a fundamentação das análises aqui propostas; e por último são apresentadas as conclusões da discussão teórica sobre políticas públicas e os dados estatísticos coletados referentes à gestão no município de Campos dos Goytacazes, tendo como base de referência comparativa para tais conclusões os dados coletados nos dois anos referidos (2010/11 e 2014/15).

Esse recorte temporal deu-se, principalmente, por corresponder ao período de grande arrecadação de royalties petrolíferos, seguido da transição econômica afetada pela política de redistribuição destes, conforme Lei 12.734, de 30 de novembro de 2012, e, assim, proporcionar uma análise do cenário em que se encontra, nos últimos cinco anos, a gestão cultural no município.

A metodologia aplicada corresponde ao levantamento bibliográfico dos conceitos abordados: políticas públicas, gestão cultural; a formatação do banco de dados dos eventos culturais pesquisados e estatisticamente elencados; e a análise desses dados é feita mediante os conceitos teóricos abordados, tecendo, por fim, considerações sobre a atual gestão cultural no município de Campos dos Goytacazes.

\section{Referencial teórico}

\section{Políticas Públicas}

Ao reportar-se à política pública como área de conhecimento e disciplina acadêmica, encontra-se sua gênese nos EUA, em um 
processo de rompimento com as etapas seguidas pela tradição europeia de estudos e pesquisas nessa área, que se concentravam na análise muito mais sobre o Estado e suas instituições do que na produção de governos:

O pressuposto analítico que regeu a constituição e a consolidação dos estudos sobre políticas públicas é o de que, em democracias estáveis, aquilo que o governo faz ou deixa de fazer é passível de ser (a) formulado cientificamente e (b) analisado por pesquisadores independentes. A trajetória da disciplina, que nasce como subárea da ciência política, abre o terceiro grande caminho trilhado pela ciência política norte-americana no que se refere ao estudo do mundo público. $\mathrm{O}$ primeiro, seguindo a tradição de Madison, cético da natureza humana, focalizava o estudo das instituições, consideradas fundamentais para limitar a tirania e as paixões inerentes à natureza humana. $\mathrm{O}$ segundo caminho seguiu a tradição de Paine e Tocqueville, que viam, nas organizações locais, a virtude cívica para promover o "bom" governo. O terceiro caminho foi o das políticas públicas como um ramo da ciência política para entender como e por que os governos optam por determinadas ações. (SOUZA, 2006, p.22).

Segundo as considerações de Souza (2006), o aprimoramento do conceito de políticas públicas deve-se aos autores Laswell, Simon, Lindblom e a Easton. Sendo que o primeiro introduziu a expressão policy analysis (análise de política pública) como forma de conciliar conhecimento científico/acadêmico com a produção empírica dos governos e também como forma de estabelecer o diálogo entre cientistas sociais, grupos de interesse e governo.

$\mathrm{O}$ segundo autor trabalhou o conceito de racionalidade limitada dos decisores públicos (policy makers), que se poderia traduzir como gestor político ou aquele que tem o poder de decisão sobre as ações políticas de cada setor. Tal conceito mostra que a limitação da racionalidade poderia ser minimizada pelo conhecimento racional. Considera, ainda, que a racionalidade dos gestores públicos é sempre limitada por problemas tais como: informação incompleta ou imperfeita; tempo para a tomada de decisão; autointeresse dos gestores. Porém tais ações podem ser maximizadas até um ponto satisfatório pela criação de estruturas (conjunto de regras, instituições e recursos) que enquadrem o comportamento dos atores e modelem esse comportamento na direção de resultados desejados, impedindo, inclusive, a busca de maximização de interesses próprios. 
Já o terceiro, Lindblom, questionou a ênfase no racionalismo de Laswell e Simon, propondo a incorporação de outras variáveis à formulação e à análise de políticas públicas, tais como as relações de poder e a integração entre as diferentes fases do processo decisório, o que não teria necessariamente um fim ou um princípio. Considera-se, assim, que as políticas públicas precisariam incorporar outros elementos à sua formulação e à sua análise, além das questões de racionalidade, tais como o papel das eleições, das burocracias, dos partidos e dos grupos de interesse.

Por último, Easton contribuiu para a área ao definir a política pública como um sistema, ou seja, como uma relação entre formulação, resultados e o ambiente, onde políticas públicas recebem inputs dos partidos, da mídia e dos grupos de interesse que influenciam seus resultados e efeitos (SOUZA, 2006).

Dessa forma, a política encontra-se expressa em três vertentes: quem pensa (Policy Thinks), quem faz (Policy Maker) e quem recebe (Policy Taker). Política pública, portanto, pode ser definida como um campo dentro do estudo da política que analisa o governo à luz de grandes questões públicas, como um conjunto de ações de Estado, e não apenas de governo, que irão produzir efeitos específicos, sendo a soma das ações - por meio de atividades específicas - que agem diretamente ou por meio de delegação e que influenciam a vida dos cidadãos. Porém, a Política Pública pressupõe, ainda, a participação da sociedade civil na esfera de decisão e formulação das políticas, e não apenas na implementação e execução (DAGNINO, 2005). Ao nos determos no estudo de políticas públicas de cultura é necessário ressaltar que uma política cultural na efetivação ideológica que permeia suas ações, traz embutida uma concepção de cultura. Esclarecer o conceito de cultura imanente à política cultural deverá ser um procedimento analítico vigoroso afim de que a amplitude do conceito de cultura utilizado não apenas venha a delinear a extensão dos objetos das políticas culturais, mas comportar as questões a serem enfrentadas por tais políticas (RUBIM, 2007).

Dessa forma, não se encontrará uma política pública de cultura que se isente do conceito de cultura de onde partem suas ações políticas. Considerando, ainda, que política pública implica "colocar o governo em ação", entende-se que tais políticas traduzem os propósitos e plataformas eleitorais de governos democráticos em programas e ações que produzirão resultados ou mudanças no mundo real. 


\section{Políticas públicas na gestão de cultura}

É importante ressaltar que políticas públicas, no que se refere à gestão em cultura, perpassa, segundo Canclini (2005), por dois eixos: a) gestões culturais tradicionalistas, aquelas que promovem as práticas culturais nacionais e populares autênticas a serem preservadas da industrialização, da massificação urbana e das influências estrangeiras e b) gestões culturais que partem de uma concepção de arte pela arte, sem fronteiras territoriais, confiando na experimentação e na inovação autônoma, com fé no progresso.

Considerando os modelos ideológicos de elaboração dessas políticas, destacam-se: a) política de dirigismo cultural - podendo ter como tônica principal a cultura popular ou não; b) política de liberalismo cultural - que não defende modelos únicos de representação simbólica nem entende, necessariamente, que é dever do Estado promover a cultura e oferecer opções culturais à população; c) políticas de democratização cultural - baseadas no princípio de que a cultura é uma força social de interesse coletivo que não pode ficar à mercê das disposições ocasionais do mercado, devendo, portanto, ser apoiada em princípios consensuais.

A visão de política pública de cultura, defendida pelo Plano Nacional de Cultura em consonância com essa terceira vertente, comprometia-se em ser um Plano que efetivamente traçasse metas, estratégias, diretrizes e também executasse políticas que fomentassem o pluralismo e promovessem o acesso à produção e ao usufruto de bens e serviços culturais (PNC, 2009, p.12)

Um dos papéis dessa proposta para as políticas de cultura no Brasil refere-se à abertura do diálogo com a sociedade, em uma visão democrática e ampla no entendimento do conceito de cultura. A visão antropológica desse conceito passa a ocupar o lugar central, e as diversas modalidades de cultura a obter o olhar atento e indiscriminado dos promotores de cultura. Em uma intensa proposição de construir políticas públicas com base no debate com a sociedade é que surgem os seminários, as câmaras setoriais, as conferências, culminando com a Conferência Nacional de Cultura.

Nessa perspectiva, passa-se a compreender o desenvolvimento a partir da (re)construção de bases culturais locais. As políticas culturais começam a participar dos processos de municipalização, 
1 Informações disponíveis em: <http://blogs.cultura.gov.br/ cnc/files/2010/03/Propostas32-finais.pdf> com o objetivo de resgatar, por meio do fomento, a diversidade cultural, a capacidade de autodeterminação das comunidades, trabalhando essa diversidade a favor do desenvolvimento territorial sustentável, local e regional.

A realização da I Conferência Nacional de Cultura (13 a 16 de dezembro de 2005), a partir da qual o poder público extraiu subsídios para a confecção do Plano Nacional de Cultura, publicado em 2009, foi o momento estratégico de inserir as instâncias federativas, estaduais e municipais em um grande debate sobre os rumos das políticas públicas de cultura no Brasil.

No contexto da construção dessa Conferência, realizouse, no período de setembro a dezembro de 2005, cerca de 400 encontros municipais, intermunicipais, estaduais e setoriais, além de uma plenária nacional, com a participação de gestores de 1158 municípios, de 19 estados e do Distrito Federal e uma rede de debates que envolveram cerca de 60 mil participantes. Nessa Conferência foram desenvolvidas as bases das diretrizes gerais que compõem o projeto de lei do Plano Nacional de Cultura (PNC) (BRASIL/MinC, 2015) .

O Plano Nacional de Cultura foi apresentado em março de 2006 ao Congresso Nacional com o projeto de Lei 6.837. Debatido no Parlamento desde então, o PNC foi aprovado em 2 de dezembro de 2010 e transformado na Lei 12.343/10.

Ainda na expectativa de aprovação do PNC, foi realizada, de 11 a 14 de março de 2010, em Brasília, a II Conferência Nacional de Cultura, que, entre suas principais contribuições, marcou as 32 prioridades das ações de Cultura para o País, entre as quais se destacam os principais eixos: Eixo 1: Produção Simbólica e Diversidade Cultural; Eixo 2: Cultura, Cidade e Cidadania; Eixo 3: Cultura e Desenvolvimento Sustentável; Eixo 4: Cultura e Economia Criativa; Eixo 5: Gestão e Institucionalidade da Cultura1.

A estrutura central do PCN engloba conceitos e valores norteadores e é dividida em modalidades de ação do Estado e cinco estratégias gerais, entre as quais se destacam: a) Fortalecer a ação do Estado no planejamento e na execução das políticas culturais; b) Incentivar, proteger e valorizar a diversidade artística e cultural brasileira; c) Universalizar o acesso dos brasileiros à fruição e à produção cultural; d) Ampliar a participação da cultura no desenvolvimento socioeconômico sustentável; e) Consolidar os sistemas de participação social na gestão das políticas culturais (PNC, 2009). 
A análise das Políticas Públicas de Cultura no Município de Campos dos Goytacazes, foi norteada pelos cinco pilares em que foram construídos o PNC, considerando que o grande papel das políticas culturais, ao trabalhar a diversidade cultural, encontra-se na (re)construção e na valorização das bases locais. A partir do levantamento da oferta de cultura realizada pela Gestão Pública no período de 2010 a 2015 no Município de Campos dos Goytacazes, buscou-se compreender o sentido de tais Políticas, sua forma de gestão e seus impactos sociais.

\section{Políticas Públicas em Cultura no Município de Campos dos Goytacazes}

\section{O Município de Campos dos Goytacazes}

O Município de Campos dos Goytacazes, situado ao norte do estado do Rio de Janeiro, possui, segundo o Censo 2010, uma população de 463.731 , em uma área compreendida de $4.027 \mathrm{Km}^{2}$, na Mata Atlântica².

A formação socioespacial desse município data do período colonial brasileiro, quando ocorreu a ocupação portuguesa nas terras dos índios Goitacá, o que explica o seu topônimo atual. Da sua formação colonial até a década de 1970, a base econômica do município esteve relacionada à produção canavieira, alternando períodos de grande desenvolvimento com longos períodos de decadência.

Entretanto, a partir dos anos de 1970, a história desse município tem um divisor de águas, a saber, a instalação das bases da Petrobras no município de Macaé, que levou a região Norte Fluminense (na qual Campos encontra-se inserida) a um novo sistema produtivo.

Em 1974, a Petrobras elege a cidade de Macaé como base de operações das atividades de prospecção e de produção para o recém-descoberto petróleo da plataforma continental da Bacia de Campos, e inaugura-se um novo ciclo econômico regional, agora baseado direta e indiretamente nos recursos oriundos da exploração petrolífera. (PIQUET, 2003, p. 223).

A implantação das bases produtivas do petróleo na cidade de Macaé resultou em grande indignação por parte das autoridades e frustração para população de Campos dos Goytacazes. A partir
2 Informações disponíveis em: <http://www.ibge.gov. $\mathrm{br} /$ cidadesat/painel/painel. php? codmun=330100\#> 
de 1999, Campos tornou-se (juntamente com Macaé, Cabo Frio, Quissamã e Rio das Ostras) um dos municípios que mais recebem recursos advindos da exploração de petróleo no Brasil, o que trouxe um grande diferencial ao orçamento municipal (PIQUET, 2003).

Essa mudança substancial no orçamento do município, como demonstrado na Tabela 1 e no Gráfico 1, representou uma grande expectativa de desenvolvimento econômico local. $\mathrm{O}$ município passa a obter a atenção nacional sobre sua forma de administrar o tamanho orçamento e as perspectivas de implementação de políticas públicas que gerassem crescimento, de fato, não só nesse município, mas na região na qual encontra-se inserido.

\section{Tabela 1 - Evolução dos royalties petrolíferos de 2005 a 2015}

\begin{tabular}{|l|l|l|l|l|}
\hline Beneficiario / Estado & Ano & Royalties + Participaçöes Especiais & Receita Orçamentaria & $\begin{array}{l}\text { (Royalties + PE ) } \\
\text { Receita Orçamentaria }\end{array}$ \\
\hline CAMPOS DOS GOYTACAZES / Rio de Janeiro & 2005 & $\mathrm{R} \$ 678.425 .571,67$ & $\mathrm{R} \$ 940.689 .607,50$ & 0.721200241037 \\
\hline CAMPOS DOS GOYTACAZES / Rio de Janeiro & 2006 & $\mathrm{R} \$ 852.565 .850,92$ & $\mathrm{R} \$ 1.249 .186 .089,00$ & 0.68249707423695 \\
\hline CAMPOS DOS GOYTACAZES / Rio de Janeiro & 2007 & $\mathrm{R} \$ 780.099 .183,21$ & $\mathrm{~N} / \mathrm{D}$ & $\mathrm{N} / \mathrm{D}$ \\
\hline CAMPOS DOS GOYTACAZES / Rio de Janeiro & 2008 & $\mathrm{R} \$ 1.168 .642 .499,45$ & $\mathrm{~N} / \mathrm{D}$ & $\mathrm{N} / \mathrm{D}$ \\
\hline CAMPOS DOS GOYTACAZES / Rio de Janeiro & 2009 & $\mathrm{R} \$ 993.167 .279,11$ & $\mathrm{R} \$ 1.423 .568 .588,12$ & 0.69766029357363 \\
\hline CAMPOS DOS GOYTACAZES / Rio de Janeiro & 2010 & $\mathrm{R} \$ 1.016 .021 .871,72$ & $\mathrm{R} \$ 1.867 .225 .055,18$ & 0.54413466062989 \\
\hline CAMPOS DOS GOYTACAZES / Rio de Janeiro & 2011 & $\mathrm{R} \$ 1.235 .611 .249,66$ & $\mathrm{R} \$ 2.044 .461 .698,14$ & 0.60436996730441 \\
\hline CAMPOS DOS GOYTACAZES / Rio de Janeiro & 2012 & $\mathrm{R} \$ 1.354 .233 .313,47$ & $\mathrm{R} \$ 2.403 .732 .736,53$ & 0.56338763993578 \\
\hline CAMPOS DOS GOYTACAZES / Rio de Janeiro & 2013 & $\mathrm{R} \$ 1.303 .272 .971,54$ & $\mathrm{~N} / \mathrm{D}$ & $\mathrm{N} / \mathrm{D}$ \\
\hline CAMPOS DOS GOYTACAZES / Rio de Janeiro & 2014 & $\mathrm{R} \$ 1.208 .366 .996,05$ & $\mathrm{~N} / \mathrm{D}$ & $\mathrm{N} / \mathrm{D}$ \\
\hline CAMPOS DOS GOYTACAZES / Rio de Janeiro & 2015 & $\mathrm{R} \$ 371.871 .222,68$ & $\mathrm{~N} / \mathrm{D}$ & $\mathrm{N} / \mathrm{D}$ \\
\hline
\end{tabular}

Fonte: http://inforoyalties.ucam-campos.br

\section{Gráfico 1 - Evolução dos royalties petrolíferos de 2005 a 2015}

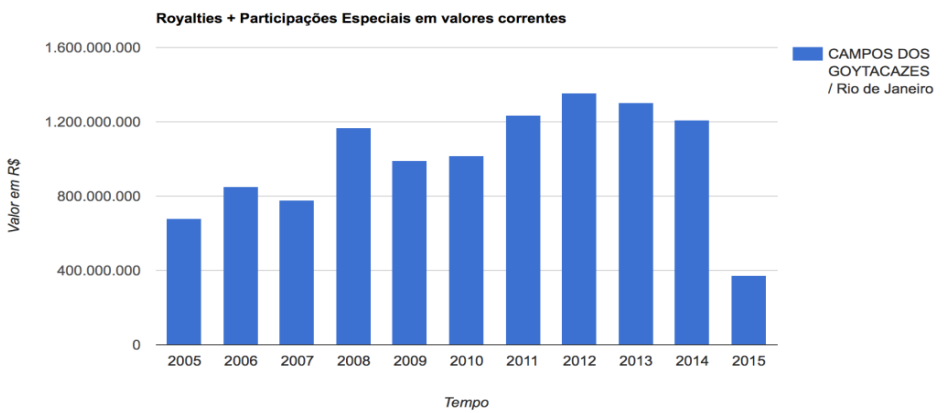

Fonte: http://inforoyalties.ucam-campos.br 
Tabela 2 - Evolução do orçamento de Campos dos Goytacazes e os investimentos em cultura de 1999 a 2013

\begin{tabular}{|c|c|c|c|c|c|}
\hline Ano de Exercício & Orçamento Inicial & Receita Total & $\begin{array}{l}\text { Despesas totais } \\
\text { empenhadas }\end{array}$ & $\begin{array}{c}\text { Despesas } \\
\text { empenhadas com } \\
\text { cultura }\end{array}$ & $\begin{array}{c}\text { \% Em } \\
\text { relação ao } \\
\text { total }\end{array}$ \\
\hline $1999^{*}$ & $95.000 .000,00$ & SR & SR & SR & $\mathrm{SR}$ \\
\hline 2000 & $144.160 .000,00$ & SR & SR & SR & SR \\
\hline $2001^{*}$ & $228.925 .100,00$ & SR & $S R$ & SR & SR \\
\hline $2002^{*}$ & $434.257 .950,00$ & SR & SR & SR & SR \\
\hline 2003 & $450.342 .466,00$ & SR & SR & SR & $\mathrm{SR}$ \\
\hline 2004 & $600.000 .000,00$ & $818.195 .414,45$ & $821.820 .405,29$ & $11.439 .000,00$ & $1,39 \%$ \\
\hline 2005 & $802.016 .822,07$ & $925.509 .307,49$ & SR & SR & $\mathrm{SR}$ \\
\hline 2006 & $919.958 .351,42$ & $1.249 .186 .088,91$ & $\begin{array}{ll}\mathrm{R} \$ & 1.132 .547 .053,07 \\
\end{array}$ & $15.911 .128,94$ & $1,40 \%$ \\
\hline 2007 & $1.165 .485 .395,78$ & $1.397 .405 .495,78$ & $\mathrm{SR}$ & $S R$ & $\mathrm{SR}$ \\
\hline $2008^{*}$ & $1.452 .184 .172,00$ & $1.130 .279 .343,56$ & SR & SR & SR \\
\hline 2009 & $1.545 .075 .126,00$ & 1.423 .568 .588 .12 & $\begin{array}{ll}R \$ & 1.239 .293 .790,26\end{array}$ & SR & $S R$ \\
\hline 2010 & $1.413 .407 .262,50$ & $1.867 .225 .055,18$ & $\begin{array}{ll}\mathrm{R} \$ & 1.876 .758 .880,03\end{array}$ & $20.607 .616,16$ & $1,10 \%$ \\
\hline 2011 & $1.876 .789 .985,59$ & $2.110 .358 .531,91$ & $1.952 .040 .264,34$ & $19.625 .864,80$ & $1,01 \%$ \\
\hline 2012 & $2.195 .709 .484,87$ & $2.403 .732 .736,53$ & $2.200 .102 .483,39$ & SR & $S R$ \\
\hline 2013 & $2.410 .000 .000,00$ & $2.532 .588 .436,87$ & $2.297 .568 .901,99$ & $28.574 .175,40$ & $1,24 \%$ \\
\hline
\end{tabular}

Fonte: Tribunal de Contas do Estado do Rio de Janeiro. Disponível em: $<$ http://www.tce.ri.gov.br>

$\mathrm{Na}$ Tabela 2, pode-se observar o demonstrativo do crescimento orçamentário do município e os investimentos para a área cultural. Os valores percentuais referentes às despesas empenhadas com cultura foram mantidos em $1,01 \%$ a $1,39 \%$ do orçamento total, não trazendo, no entanto, aumento proporcional à receita. Embora o quantitativo de $\mathrm{R} \$ 28$ milhões, o mais alto conferido para aplicação de recursos em políticas culturais no ano de 2013, corresponda a 60\% do orçamento total de alguns municípios do Estado do Rio de Janeiro, tais como Cardoso Moreira, Aperibé, Sumidouro ${ }^{3}$, dentre outros, é importante ressaltar que o município de Campos dos Goytacazes não sofre restrição orçamentária, considerando o volume de recebimentos em royalties petrolíferos e as participações especiais, colocando-o dentre os municípios mais "ricos" do Brasil, apresentado,em 2011, como o $13^{\circ}$ município no ranking dos maiores municípios do Brasil no PIB ${ }^{4}$. No entanto, a aplicação desses recursos só poderá ser analisada a partir do levantamento da oferta disponibilizada pela gestão cultural do município que apresentar-se-á a seguir nas tabelas 3 e 4 .
3 Informações disponíveis em: < http://www.tce.rj.gob.br >

4 Informação disponível em: $<$ http://www.economia.terra. com.br/pibs-municipio/> 
Tabela 3 - Despesas do Município de Campos dos Goytacazes no ano de 2010

\begin{tabular}{|c|c|c|}
\hline FUNÇÃO & $\begin{array}{c}\text { DESPESA } \\
\text { EMPENHADA }(\mathrm{R} \$)\end{array}$ & $\begin{array}{c}\text { \% EM RELAÇÃO } \\
\text { AO TOTAL }\end{array}$ \\
\hline 1.Administração & $506.662 .403,74$ & $27,00 \%$ \\
\hline 2.Saúde & $438.344 .819,26$ & $23,35 \%$ \\
\hline 3.Educação & $237.977 .547,39$ & $12,67 \%$ \\
\hline 4.Urbanismo & $230.379 .045,53$ & $12,28 \%$ \\
\hline 5.Habitação & $202.468 .544,25$ & $10,79 \%$ \\
\hline $\begin{array}{l}\text { 6.Gestão } \\
\text { Ambiental }\end{array}$ & $64.855 .926,96$ & $3,46 \%$ \\
\hline $\begin{array}{l}\text { 7.Assistência } \\
\text { Social }\end{array}$ & $51.755 .454,43$ & $2,76 \%$ \\
\hline 8.Transporte & $34.569 .191,01$ & $1,84 \%$ \\
\hline $\begin{array}{l}\text { 9.Encargos } \\
\text { Especiais }\end{array}$ & $26.328 .614,88$ & $1,40 \%$ \\
\hline 10.Cultura & $20.607 .616,16$ & $1,10 \%$ \\
\hline 11.Saneamento & $19.391 .756,24$ & $1,03 \%$ \\
\hline 12.Legislativo & $16.195 .629,63$ & $0,86 \%$ \\
\hline 13.Judiciário & $7.235 .909,10$ & $0,39 \%$ \\
\hline 14.Agricultura & $6.332 .079,59$ & $0,34 \%$ \\
\hline $\begin{array}{l}\text { 15.Desporto e } \\
\text { Lazer }\end{array}$ & $4.304 .185,77$ & $0,23 \%$ \\
\hline $\begin{array}{l}\text { 16.Direitos da } \\
\text { Cidadania }\end{array}$ & $3.718 .104,57$ & $0,20 \%$ \\
\hline 17.Indústria & $3.165 .152,35$ & $0,17 \%$ \\
\hline $\begin{array}{l}\text { 18.Segurança } \\
\text { Pública }\end{array}$ & $1.338 .463,75$ & $0,07 \%$ \\
\hline $\begin{array}{l}\text { 19.Comércio e } \\
\text { Serviços }\end{array}$ & $842.279,41$ & $0,04 \%$ \\
\hline $\begin{array}{l}\text { 20.Previdência } \\
\text { Social }\end{array}$ & $285.760,01$ & $0,02 \%$ \\
\hline $\begin{array}{l}\text { 21.Ciência e } \\
\text { Tecnologia }\end{array}$ & 396,00 & $0,00 \%$ \\
\hline Total & $1.876 .758 .880,03$ & $100,00 \%$ \\
\hline
\end{tabular}

Fonte: Tribunal de Contas do Estado do Rio de Janeiro (TCE-RJ), processo no 206.1259/11 rubrica fls.: 1908.

Como pode ser visto na Tabela 3, o investimento em cultura, no ano de 2010, chegou a $\mathrm{R} \$ 20.607 .616,16$, perfazendo $1,10 \%$ de todo orçamento do município. Já no ano de 2013, o orçamento 
passou para $\mathrm{R} \$ 28.548 .127,08$, percentual de 1,24 de todo o orçamento (Tabela 4). Em virtude da prestação de contas da administração financeira do exercício do ano de 2014 não estar disponível, não foi possível comparar os valores referentes a esse período. Vale ressaltar, no entanto, que devido à redistribuição de royalties e participações especiais, o orçamento de 2015 teve uma queda significativa para 371.871.222,68, como pode ser visto no Gráfico 1. Tal política trouxe consequências para o município como um todo, e principalmente para o setor cultural, que, como pode ser visualizado no levantamento apresentado, teve uma redução considerável de suas atividades no âmbito da cultura.

Tabela 4 - Despesas do Município de Campos dos Goytacazes no ano de 2013

\begin{tabular}{|c|l|r|r||}
\hline CÓDIGO & \multicolumn{1}{|c|}{ FUNÇÃO } & DESPESA EMPENHADA RS & $\begin{array}{c}\text { \% EM RELAÇÃO AO } \\
\text { TOTAL }\end{array}$ \\
\hline \hline 10 & Saúde & $629.399 .492,56$ & $27,40 \%$ \\
\hline 4 & Administração & $618.626 .165,00$ & $26,93 \%$ \\
\hline 12 & Educação & $337.540 .300,86$ & $14,69 \%$ \\
\hline 15 & Urbanismo & $298.331 .254,73$ & $12,98 \%$ \\
\hline 16 & Habitação & $97.569 .116,39$ & $4,25 \%$ \\
\hline 18 & Gestão Ambiental & $78.151 .326,23$ & $3,40 \%$ \\
\hline 8 & Assistência Social & $58.335 .558,02$ & $2,54 \%$ \\
\hline 26 & Transporte & $52.172 .654,62$ & $2,27 \%$ \\
\hline 13 & Cultura & $28.574 .175,40$ & $1,24 \%$ \\
\hline
\end{tabular}

\begin{tabular}{||c|l|r|c||}
\hline 1 & Legislativa & $27.490 .438,57$ & $1,20 \%$ \\
\hline 17 & Saneamento & $21.498 .896,53$ & $0,94 \%$ \\
\hline 28 & Encargos Especiais & $13.797 .173,30$ & $0,60 \%$ \\
\hline 2 & Judiciária & $9.761 .403,64$ & $0,42 \%$ \\
\hline 22 & Indústria & $6.435 .233,26$ & $0,28 \%$ \\
\hline 14 & Direitos da Cidadania & $6.392 .979,61$ & $0,28 \%$ \\
\hline 27 & Desporto e Lazer & $6.226 .585,14$ & $0,27 \%$ \\
\hline 20 & Agricultura & $4.708 .555,61$ & $0,20 \%$ \\
\hline 23 & Comércio e Serviços & $1.392 .896,28$ & $0,06 \%$ \\
\hline 6 & Segurança Pública & $1.003 .449,06$ & $0,04 \%$ \\
\hline 9 & Previdência Social & $130.837,82$ & $0,01 \%$ \\
\hline 25 & Energia & $30.409,36$ & $0,00 \%$ \\
\hline \hline & TOTAL & $2.297 .568 .901,99$ & $100,00 \%$ \\
\hline
\end{tabular}

Fonte: Tribunal de Contas do Estado do Rio de Janeiro (TCE-RJ), processo $\mathrm{n}^{\circ}$ 207.880-4/2014 rubrica fls.: 1708/09.

\section{A Gestão Pública de Cultura em Campos dos Goytacazes}

O município de Campos dos Goytacazes teve o seu setor de cultura, a partir dos anos 90, estruturado, primeiramente, em três Fundações Culturais: a) Fundação Cultural Jornalista Oswaldo 

e, a partir de então, a gestão de cultura do município, além das três fundações já mencionadas, passaram a contar com mais esse órgão gestor de cultura.

O município de Campos dos Goytacazes realizou, no período de 22 a 24 de setembro de 2006, a I Conferência Municipal de Cultura. Como resultado dos debates, três eixos principais concentraram as propostas dos participantes da conferência: Gestão Pública, Papel do Conselho Municipal e Empreendedorismo Cultural. Um dos itens elencados na proposta de Gestão Pública requeria a implantação do Conselho Municipal de Cultura.

Em 9 de julho de 2007, por meio da Lei Municipal 7.919, é criado o Conselho Municipal de Cultura e são designados seus representantes, seu funcionamento e atribuiçõos ${ }^{5}$. Porém, somente

5 Informações disponíveis em: http://www.campos. rj.gov.br/leis/2007/Lei\%20 Municipa1\%207.919\%20 de\%202007.pdf

6 Informações disponíveis em: http://comculturacamposrjleis. blogspot.com/2009/02/ membros-do-conselhomunicipal-de.html

7 Informações disponíveis em: http://www.campos.rj.gov. br/exibirNoticia.php?id_ noticia $=886$ por meio da Portaria no 1.840/2008, publicada em 7 de agosto de 2008, foram nomeados os membros Titulares e Suplentes até 31 de dezembro de 2008 . $^{6}$

Em um processo de descontinuidade e imobilidade, o Conselho não apresenta participação no ano de 2009, até que são nomeados novos membros pela portaria de 31 de dezembro de 2009, os quais tomaram posse em 29 de março de $2010^{7}$. Dentro dessa mesma expectativa foi criado o Comitê Gestor do Fundo Municipal de Cultura (Funcultura), que foi empossado em 2 de julho de 2011.

No processo de Gestão de Cultura, o município realizou, de 13 a 15 de setembro de 2012, a II Conferência Municipal de Cultura com objetivo de debater o futuro Plano Municipal de Cultura. A III Conferência Municipal de Cultura ocorreu em 20 de julho de 2013, teve como objetivo reunir pensamentos, demandas, propostas e necessidades da população de Campos dos Goytacazes, contribuindo para a realização de um amplo diagnóstico de sua diversidade cultural. A IV Conferência, realizada em 26 e 27 de setembro de 2014, teve objetivo de debater a composição do Plano Decenal de Cultura no Município.

$\mathrm{O}$ que pode-se observar na gestão de cultura durante o período da realização deste estudo, isto é, de 2010 a 2015, foi a extinção da Secretaria Municipal de Cultura e das Fundações de Cultura, com a alegação de contenção de gastos devido à queda no orçamento. Tais modificações visavam "enxugar" a máquina 
administrativa. O Executivo Municipal passa a contar com dez secretarias; 26 superintendências, para onde foram deslocados os demais secretários ${ }^{8}$.

Dessa forma, a gestão municipal para assuntos relacionados à cultura ficam com a Secretaria de Educação, Cultura e Esportes, representados pelas: Superintendência de Igualdade Racial; Superintendência da Fundação Jornalista Oswaldo Lima; e Superintendência da Fundação de Esportes.

Assim, as três Fundações Culturais, Fundação Cultural Jornalista Oswaldo Lima; Fundação Trianon e Fundação Zumbi dos Palmares, e a Secretaria Municipal de Cultura deixam de existir, concentrando toda a Gestão em Cultura do Município na Superintendência da Fundação Jornalista Oswaldo Lima, a qual se torna o único órgão administrativo para a implementação de toda e qualquer política pública relacionada à cultura no Município.

\section{Oferta de eventos culturais apresentada pela gestão municipal de cultura no período de fevereiro de 2010 a janeiro de 2011}

A primeira parte desse levantamento foi realizado durante 12 meses, compreendidos entre fevereiro de 2010 a janeiro de 2011, no qual foram registrados todos os dados da oferta de cultura disponibilizada à população do município de Campos dos Goytacazes. Na impossibilidade da pesquisa ser feita in loco, foram utilizadas as informações disponíveis no site oficial da Prefeitura Municipal de Campos dos Goytacazes ${ }^{9}$.

O levantamento completo constou de um quantitativo de 619 ocorrências, as quais foram selecionadas considerando suas especificidades e classificação entre as diversas expressões culturais (ROCHA, 2011). Partindo desse pressuposto para tornar possível a análise das políticas públicas existentes no município, foi realizada uma primeira classificação, dividindo esses eventos pela natureza de sua ação, como pode ser visto a seguir no Quadro 1.
8 Informações disponíveis em:

< http://www.campos.rj.gov. br>
9 Informações disponíveis em: $<$ http://www.campos.rj.gov.br> 
Quadro 1 - Classificação e quantitativo dos eventos culturais ofertados pela prefeitura de Campos dos Goytacazes no período de fevereiro de 2010 a janeiro de 2011

\begin{tabular}{|c|c|c|}
\hline Área & $\begin{array}{l}\mathrm{n}^{\circ} \text { de eventos } \\
\text { promovidos }\end{array}$ & Porcentagem \\
\hline Arquitetura & 1 & $0 \%$ \\
\hline Artes plásticas & 18 & $3 \%$ \\
\hline Artesanato & 14 & $2 \%$ \\
\hline Atividades natalinas & 10 & $2 \%$ \\
\hline Carnaval & 34 & $5 \%$ \\
\hline Cinema & 25 & $4 \%$ \\
\hline Circo & 2 & $0 \%$ \\
\hline Conferência em cultura & 2 & $0 \%$ \\
\hline Cultura afro brasileira & 26 & $4 \%$ \\
\hline Cultura indigena & 1 & $0 \%$ \\
\hline Cultura religiosa & 3 & $0 \%$ \\
\hline Curso de artes & 1 & $0 \%$ \\
\hline Dança & 10 & $2 \%$ \\
\hline Desfile cívico & 1 & $0 \%$ \\
\hline Escola de musica & 3 & $0 \%$ \\
\hline Exposição & 12 & $2 \%$ \\
\hline Financiamento cultural & 4 & $1 \%$ \\
\hline Folclore & 1 & $0 \%$ \\
\hline Gastronomia & 2 & $0 \%$ \\
\hline Gestão em cultura & 18 & $3 \%$ \\
\hline infraestrutura de cultura & 11 & $2 \%$ \\
\hline Literatura & 55 & $9 \%$ \\
\hline Manifestação religiosa & 8 & $1 \%$ \\
\hline Memorial cultural & 4 & $1 \%$ \\
\hline Moda & 4 & $1 \%$ \\
\hline Multicultural & 77 & $12 \%$ \\
\hline Museu & 1 & $0 \%$ \\
\hline Música & 128 & $21 \%$ \\
\hline Oficina de musica & 1 & $0 \%$ \\
\hline Patrimônio histórico & 27 & $4 \%$ \\
\hline Pesquisa de cultura & 1 & $0 \%$ \\
\hline Rancheirada & 2 & $0 \%$ \\
\hline Show pirotécnico & 1 & $0 \%$ \\
\hline Teatro & 47 & $8 \%$ \\
\hline Turismo & 8 & $1 \%$ \\
\hline Vl Bienal do Livro & 56 & $9 \%$ \\
\hline Total & 619 & $100 \%$ \\
\hline
\end{tabular}

Fonte: ROCHA (2011) 
Tabela 5 - Percentual da oferta de cultura realizada pelo Município de Campos dos Goytacazes no período de fevereiro de 2010 a janeiro de 2011, por classificação nas diversas áreas culturais

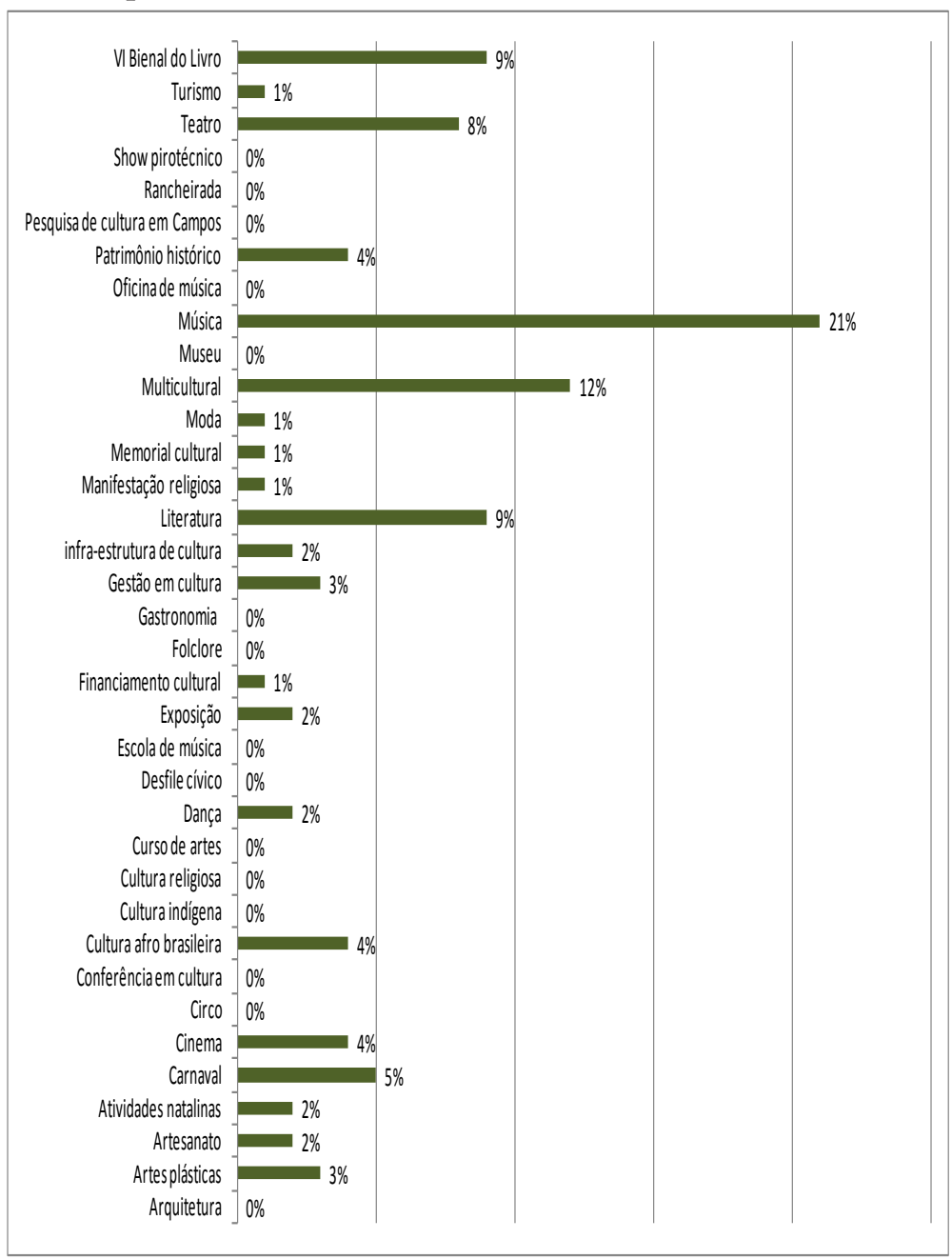

Fonte: ROCHA(2011).

Ao analisar os dados da Tabela 5 por meio do percentual indicado nas diferentes categorias de oferta de cultura, destacamse os itens que apresentam um percentual acima de $5 \%$ de ocorrência. Dentre eles tem-se: o carnaval com 5\%, o teatro com $8 \%$, a literatura com 9\%, a VI Bienal do Livro com 9\%, Multicultural com $12 \%$ e música com $21 \%$.

Diante desses percentuais, visualizam-se as atividades que foram foco da oferta de cultura no município de Campos dos 
Goytacazes, no período de 12 meses. Vale ressaltar que o período de levantamento de dados correspondeu a realização da VI Bienal do livro, o que trouxe uma dinâmica de atividades culturais que não se realizam com periodicidade, já que a bienal representa uma atividade pontual, realizada a cada dois anos.

Gráfico 2 - Percentual das atividades musicais por especificidade de estilos com desdobramento do item música apresentado na Tabela 4

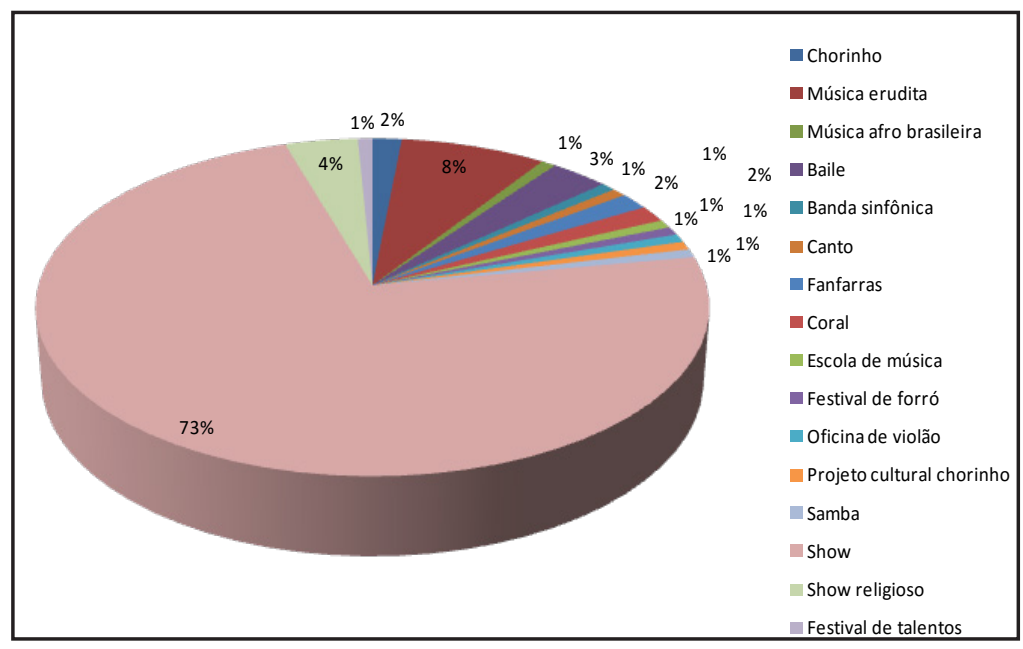

Fonte: ROCHA, 2011

Oferta de eventos culturais apresentada pela gestão municipal de cultura no período de fevereiro de 2014 a janeiro de 2015

Como pode ser observado no Quadro 2 e no Gráfico 2, a oferta de cultura realizada pela Gestão Pública do Município no período 2014/2015 demonstra que o maior percentual continua sendo na categoria shows, com carnaval com 34\%, seguido de atividades de música com $25 \%$. O levantamento completo constou de um quantitativo de 151 ocorrências, as quais foram selecionadas considerando suas especificidades e classificação entre as diversas expressões culturais. 
Quadro 2 - Classificação e quantitativo dos eventos culturais ofertados pela prefeitura de Campos dos Goytacazes no período de fevereiro de 2014 a janeiro de 2015

\begin{tabular}{|l|r|r|}
\hline \multicolumn{1}{|c|}{ Área } & $\begin{array}{c}\text { n }{ }^{\circ} \text { de } \\
\text { eventos } \\
\text { promovidos }\end{array}$ & \multicolumn{1}{|c|}{$\%$} \\
\hline Arquitetura & 0 & $0 \%$ \\
\hline Artes plásticas & 0 & $0 \%$ \\
\hline Artesanato & 4 & $3 \%$ \\
\hline Atividades natalinas & 0 & $0 \%$ \\
\hline Carnaval & 51 & $34 \%$ \\
\hline Cinema & 3 & $2 \%$ \\
\hline Circo & 0 & $0 \%$ \\
\hline Conferência em cultura & 0 & $0 \%$ \\
\hline Cultura & 0 & $0 \%$ \\
\hline Curso de artes & 0 & $0 \%$ \\
\hline Dança & 5 & $3 \%$ \\
\hline Desfile cívico & 0 & $0 \%$ \\
\hline Escola de música & 0 & $0 \%$ \\
\hline Exposição & 0 & $0 \%$ \\
\hline Financiamento cultural & 0 & $0 \%$ \\
\hline Folclore & 0 & $0 \%$ \\
\hline Gastronomia & 3 & $2 \%$ \\
\hline Gestão em cultura & 0 & $0 \%$ \\
\hline Infraestrutura em cultura & 0 & $0 \%$ \\
\hline Literatura & 13 & $9 \%$ \\
\hline Manifestação religiosa & 0 & $0 \%$ \\
\hline Memorial cultural & 0 & $100 \%$ \\
\hline Moda & 0 & $0 \%$ \\
\hline Multicultural & 0 & $0 \%$ \\
\hline Museu & 0 & $0 \%$ \\
\hline Música & 0 & $0 \%$ \\
\hline Oficina de Música & 0 & $0 \%$ \\
\hline Patrimônio histórico & 0 & $0 \%$ \\
\hline Pesquisa em cultura & 0 & $0 \%$ \\
\hline Rancheirada & 6 & $4 \%$ \\
\hline Show pirotécnico & 0 & $17 \%$ \\
\hline Teatro & 0 & 0 \\
\hline Turismo & 0 & 0 \\
\hline Total & 0 & 0 \\
\hline
\end{tabular}

Fonte: Elaborado pelo autor André Luís Almeida Peixoto com base em dados obtidos em: http://www.campos.rj.gov.br 
Gráfico 3 - Classificação e quantitativo dos eventos culturais ofertados pela prefeitura de Campos dos Goytacazes no período de fevereiro de 2014 a janeiro de 2015

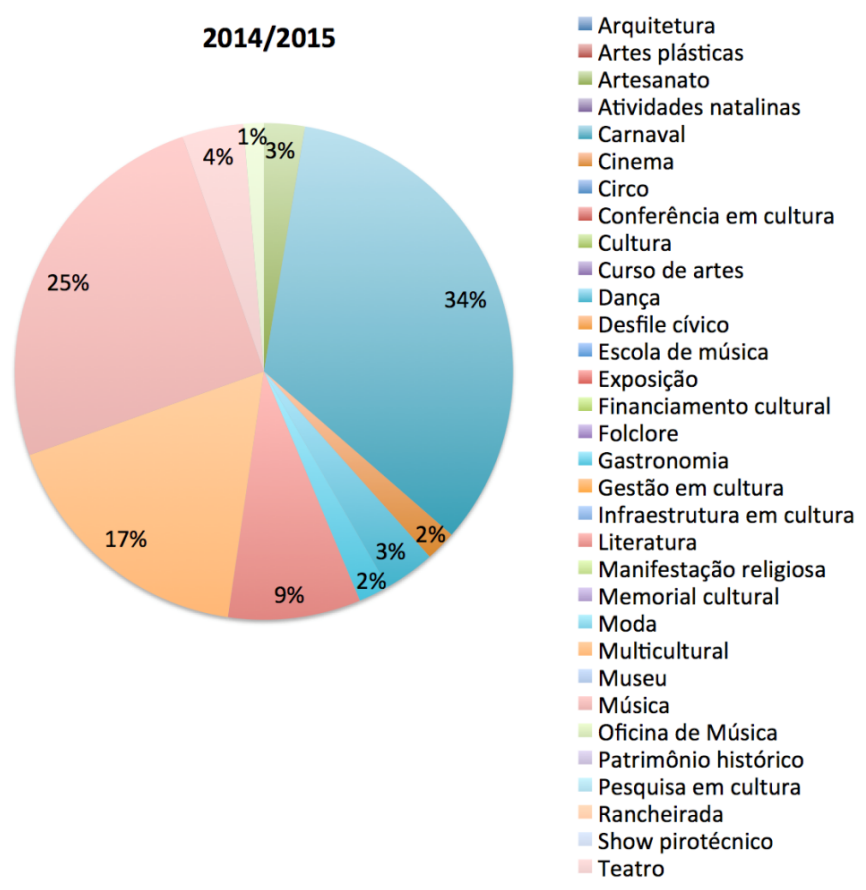

Fonte: Elaborado pelos autores (2015)

Dados comparativos entre a oferta de eventos culturais apresentada pela gestão municipal de cultura nos dois períodos (2010/11 e 2014/15)

Gráfico 4 - Quantidade de eventos em números reais comparando os dois períodos - 2010/11 e 2014/15.

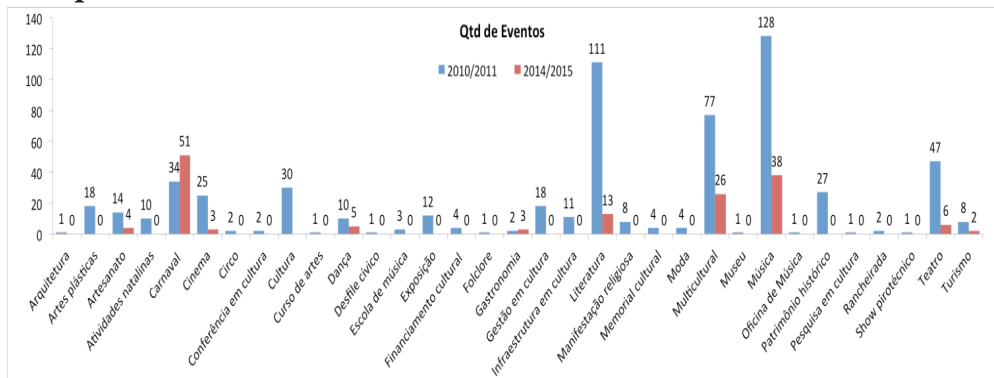

Fonte: Elaborado pelos autores (2015) 
Gráfico 5 - Porcentagem de eventos considerando a comparação entre os dois períodos - 2010/11 e 2014/15

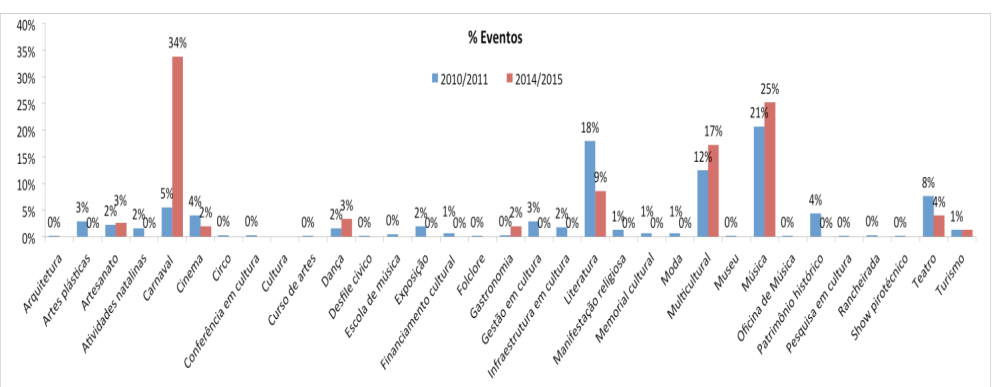

Fonte: Elaborado pelos autores (2015)

A comparação em números reais e percentuais do levantamento efetuado nos dois períodos do recorte temporal proposto neste estudo, a saber, fevereiro de 2010 a janeiro de 2011 e fevereiro de 2014 a janeiro de 2015, tornou possível demonstrar o crescimento e decrescimento das áreas culturais que receberam maior e menor proposição de atividades culturais, respectivamente. As ações elencadas na categoria música correspondem vigorosamente a apresentação de shows, principalmente nos períodos de dezembro a fevereiro, em que políticas culturais são deslocadas para o distrito de Farol de São Thomé, região litoral do município, com o Projeto "Verão da Família". As demais categorias apresentadas são pontuais, a saber, período de Festa do "São Salvador", padroeiro do município, no mês de agosto, carnaval fora de época, que acontece no mês de abril ${ }^{10}$, e literatura, que teve o percentual de $18 \%$ no levantamento do primeiro período por ter sido ano de realização da Bienal do Livro. Assim sendo, torna-se fundamental considerar as categorias que foram apresentadas como ações das políticas públicas de cultura para ser possível afirmar que a gestão de cultura do município de Campos dos Goytacazes desprivilegia atividades culturais a médio e longo prazo como proposta de formação cultural educativa e continuada para os cidadãos campistas.

\section{Considerações finais}

Considerando os aspectos apresentados em relação ao conceito de Políticas Públicas e o estudo sobre a Gestão em Cultura em Campos dos Goytacazes, concluí-se que o município
10 Informações disponíveis em: <http://www.campos. rj.gov.br $>$ 
sofre um declínio considerável das atividades culturais, embora continue contanto com os benefícios, como os royalties e o Funcultura, sendo que não disponibiliza com transparência a utilização de tais orçamentos.

Uma Gestão que antes contava com quatro órgãos gestores, como já citado, as três fundações mais a Secretaria de Cultura, na atualidade, conta com apenas a Superintendência Jornalista Oswaldo Lima. O que leva a considerar que o poder de ação centralizou-se e ainda que sejam realizadas Conferências de Cultura para o debate democrático, as ações debatidas não tiveram “eco" na implementação de Políticas Públicas para o Município.

Os resultados obtidos no levantamento da oferta de cultura no município no primeiro período, a saber, de fevereiro de 2010 a janeiro de 2011 e no segundo período, realizado de fevereiro de 2014 a janeiro de 2015 permitiram efetuar uma análise comparativa sobre as ofertas e políticas culturais implementadas nos dois períodos, considerando as semelhanças e/ou diferenças que particularizaram e acentuaram cada um desses dois momentos.

Por meio de uma análise comparativa foi possível compreender que o modelo de políticas públicas que envolvem a cultura no município baseiam-se principalmente em shows, em sua maioria "exportados", não dispondo de uma política que envolva a participação da sociedade como processo educativo, como desenvolvimento da criatividade, oportunizando aos artistas locais um espaço para exposição de seus trabalhos nas mais diversas áreas artísticas. Tais resultados permitiram observar como as políticas culturais, assim como as políticas de educação, saúde, habitação, trabalho, entre outros domínios da vida humana, merecem o debate público e político no Município de Campos dos Goytacazes.

Ainda foi relevante na comparação dos dados obtidos observar a oferta de cultura no período de 2014/2015, que revelou uma queda considerável das atividades relacionadas no período anterior, a saber, 2010/2011. E, ainda, a predominância em shows e eventos musicais como atividade de cultura ofertada.

Considera-se, assim, que as políticas culturais podem e devem qualificar as políticas de educação, saúde, habitação, trabalho, entre outros domínios da vida humana, porém não se pode deixar de observar que os debates acerca do desenvolvimento crescem com as discussões sobre a economia da cultura e seu significado estratégico para o desenvolvimento local e regional. 
Essas conquistas são importantes e passam a legitimar as políticas culturais.

Dessa forma, a cultura não deve ser somente considerada como política, a partir de seus impactos relativos à geração de empregos ou à redistribuição de renda. É fundamental considerar, nas políticas públicas culturais, o papel da cultura como um fim em si mesmo, ou seja, a cultura é, por si, responsável pela sua própria sustentabilidade. Produzir cultura, compartilhar cultura, fruir cultura são experiências únicas, fundamentais e valiosas, pois representam para o homem o substrato da sua própria existência.

\section{Referências}

BRASIL. Presidência da República. Lei 12.734, de 30 de novembro de 2012. Brasília, 2012. Disponível em: http://www.planalto.gov.br/ ccivil_03/_ato2011-2014/2012/lei/112734.htm Acesso em: 20 out. 2014.

BRASIL. Ministério da Cultura. Disponível em: <http://www.cultura. gov.br>. Acesso em: 14 mar. 2015.

CANCLINI, Nestor García. 1a Conferência Nacional de Cultura Eixos Temáticos. Ministério da Cultura, Brasília, 2005.

DAGNINO, Evelina. Políticas culturais, democracia e o projeto neoliberal. Revista Rio de Janeiro, n. 15, jan.-abr. 2005.

PIQUET, Rosélia (Org.). Petróleo, Royalties e Região. Rio de Janeiro: Garamond, 2003.

PLANO NACIONAL DE CULTURA. Diretrizes Gerais. Brasília: Ministério da Cultura, 2009.

ROCHA, E. S. Políticas Públicas de Cultura em Campos dos Goytacazes - RJ. 2011. Dissertação. (Mestrado em Planejamento Regional e Gestão de Cidades) Universidade Cândido Mendes UCAM, Campos dos Goytacazes, RJ, 2011.

RUBIM, Antonio Albino Canelas. Políticas Culturais: entre o possível e o impossível. In: NUSSBAUMER, Gisele Marchiori. Teoria e política da cultura: visões multidisciplinares. Salvador: Edufba, 2007.

SOUZA, Celina. Políticas Públicas: uma revisão da literatura. Sociologias, Porto Alegre, ano 8, no 16, jul/dez 2006, p. 20-45. 
RIO DE JANEIRO. Tribunal de contas do Estado do Rio de Janeiro. Disponível em: <http://www. tce.rj.gov.br/> Acesso em: 20 abr. 2015.

UNIVERSIDADE CÂNDIDO MENDES. Info Royalties. Disponível em: <http://inforoyalties. ucam-campos.br/> Acesso em: 27 mai. 2015.

\title{
PUBLIC POLICY AND CULTURE MANAGEMENT IN CAMPOS DOS GOYTACAZES - RJ.
}

\begin{abstract}
This article presents an analysis of public policies for culture in the city of Campos dos Goytacazes, in order to know the real culture of management of conditions in the city, as well as the relevance of such policies to society. To be considered a city with great arising economic potential of oil royalties and special participation means from there, the city enjoys a privileged budget compared to other municipalities in the country and therefore not experience a budget constraint condition. Divided into three parts correlated, this article first presents a brief history of the concept of public policy; then presents the functioning of the management culture in the city in the period 2010 to 2015; and finally presents a survey of the culture offer in the periods 2010/2011 and 2014/2015, following the closing remarks. To obtain real data on the actions taken by the city management culture during the study period, we applied a statistical methodology through a survey of the entire supply of culture held by the public administration in the period cited above. The comparative analysis of results for the periods enabled us to understand what public policy model that involves the culture in the city and find that for the most part are based mainly on creating shows, aimed at artists who mostly do not live in the city, and a lack of cultural activities that seek the participation and involvement of the local community. These results also allowed observing how cultural policies require public and political debate in the city of Campos dos Goytacazes.
\end{abstract}

Keywords: Public Politics; Culture management; Campos dos Goytacazes. 